\title{
A narrative review of MET inhibitors in non-small cell lung cancer with MET exon 14 skipping mutations
}

\author{
Mariacarmela Santarpia ${ }^{1}$, Marco Massafra ${ }^{1}$, Vittorio Gebbia ${ }^{2,3}$, Antonio D'Aquino ${ }^{1}$, Claudia Garipoli ${ }^{1}$, \\ Giuseppe Altavilla ${ }^{1}$, Rafael Rosell ${ }^{4,5,6}$ \\ ${ }^{1}$ Medical Oncology Unit, Department of Human Patology “G. Barresi”, University of Messina, Messina, Italy; ${ }^{2}$ Medical Oncology and Supportive \\ Care Unit, La Maddalena Cancer Center, Palermo, Italy; ${ }^{3}$ Department of Health Promotion, Mother and Child Care, Internal Medicine and Medical \\ Specialties, University of Palermo, Palermo, Italy; ${ }^{4}$ Catalan Institute of Oncology, Laboratory of Cellular and Molecular Biology, Institute for Health \\ Science Research Germans Trias i Pujol, Badalona, Barcelona, Spain; ${ }^{5}$ Translational Cancer Research Unit, Instituto Oncológico Dr Rosell, Dexeus \\ University Hospital, Barcelona, Spain; ${ }^{6}$ Universitat Autònoma de Barcelona, Barcelona, Spain \\ Contributions: (I) Conception and design: M Santarpia, R Rosell; (II) Administrative support: None; (III) Provision of study materials or patients: M \\ Santarpia, M Massafra, V Gebbia; (IV) Collection and assembly of data: M Santarpia, M Massafra, V Gebbia, A D'Aquino, C Garipoli, G Altavilla; (V) \\ Data analysis and interpretation: M Santarpia, R Rosell; (VI) Manuscript writing: All authors; (VII) Final approval of manuscript: All authors. \\ Correspondence to: Mariacarmela Santarpia. Medical Oncology Unit, Department of Human Patology “G. Barresi”, University of Messina, Via \\ Consolare Valeria, 1, 98125 Messina, Italy. Email: msantarpia@unime.it
}

\begin{abstract}
Treatment of advanced non-small cell lung cancer (NSCLC) has radically improved in the last years due to development and clinical approval of highly effective agents including immune checkpoint inhibitors (ICIs) and oncogene-directed therapies. Molecular profiling of lung cancer samples for activated oncogenes, including epidermal growth factor receptor $(E G F R)$, anaplastic lymphoma kinase $(A L K)$, c-ros oncogene 1 (ROS1) and $B R A F$, is routinely performed to select the most appropriate up-front treatment. However, the identification of new therapeutic targets remains a high priority. Recently, MET exon 14 skipping mutations have emerged as novel actionable oncogenic alterations in NSCLC, sensitive to MET inhibition. In this review we discuss: (I) MET gene and MET receptor structure and signaling pathway; (II) MET exon 14 alterations; (III) current data on MET inhibitors, mainly focusing on selective MET tyrosine kinase inhibitors (TKIs), in the treatment of NSCLC with MET exon 14 skipping mutations. We identified the references for this review through a literature search of papers about MET, MET exon 14 skipping mutations, and MET inhibitors, published up to September 2020, by using PubMed, Scopus and Web of Science databases. We also searched on websites of main international cancer congresses (ASCO, ESMO, IASLC) for ongoing studies presented as abstracts. MET exon 14 skipping mutations have been associated with clinical activity of selective MET inhibitors, including capmatinib, that has recently received approval by FDA for clinical use in this subgroup of NSCLC patients. A large number of trials are testing MET inhibitors, also in combinatorial therapeutic strategies, in MET exon 14-altered NSCLC. Results from these trials are eagerly awaited to definitively establish the role and setting for use of these agents in NSCLC patients.
\end{abstract}

Keywords: MET; MET exon 14 skipping mutations; MET-tyrosine kinase inhibitors (TKIs); non-small cell lung cancer (NSCLC)

Submitted Oct 13, 2020. Accepted for publication Jan 18, 2021.

doi: $10.21037 /$ tlcr-20-1113

View this article at: http://dx.doi.org/10.21037/tlcr-20-1113 


\section{Introduction}

Therapeutic management of advanced lung cancer has strikingly and ceaselessly evolved in recent years for the development and approval for clinical use of an increasing number of highly effective agents, including molecularly targeted drugs and immune checkpoint inhibitors (ICIs). Biomarker testing is routinely performed in non-small cell lung cancer (NSCLC) patients because multiple targeted therapies are currently available, including epidermal growth factor receptor (EGFR), anaplastic lymphoma kinase (ALK) and c-ros oncogene 1 (ROS1) tyrosine kinase inhibitors (TKIs) (1). The approval of the combination of dabrafenib and trametinib for $B R A F$-mutant NSCLC patients has further enlarged the number of predictive biomarkers that need to be screened at baseline to define the optimal therapeutic approach $(2,3)$. Immunotherapy has become part of the treatment for patients without driver alterations (EGFR or ALK). The ASCO and $\mathrm{OH}$ Joint Panel Guideline recommends pembrolizumab for non-squamous cell carcinoma and SCC with high PDL1 expression [tumor proportion score (TPS) $\geq 50 \%$ ] $(4,5)$. Combination of chemotherapy and immunotherapy is recommended for most patients with negative or low positive PD-L1. Recently, additional actionable oncogenic alterations other than EGFR, ALK, ROS1 and BRAF, have emerged, including MET alterations, RET and NTRK rearrangements (6-13). Herein, we describe MET exon 14 alterations and review current available data from clinical trials with MET inhibitors in MET 14 altered-NSCLC patients. Demonstration of clinical efficacy of selective MET TKIs in this subgroup of patients underlines the need to routinely include $M E T$ exon 14 mutation screening as part of the molecular testing panels performed for advanced NSCLC.

We present the following article in accordance with the Narrative Review Checklist (available at http://dx.doi. org/10.21037/tlcr-20-1113).

\section{Methods}

We performed an updated literature search for papers published up to September 2020 on the role of MET in non-small lung cancer using the following medical research databases: PubMed, Scopus and Web of Science. We also searched for abstracts on main international cancer congresses (ESMO, ASCO, IASLC) websites. We included in the search strategy the following terms: "MET", "MET deregulation", "MET and lung cancer", "MET exon 14 skipping mutations", "MET inhibitors" (see Key Search, in Appendix 1). For clinical trials, we collected and reviewed data of both completed and ongoing studies.

\section{Discussion}

\section{MET signaling pathway}

The MET receptor is encoded by the MET oncogene, located on the long arm of human chromosome 7 (7q31). This oncogene was first identified in a human osteogenic sarcoma cell line containing a transforming fusion protein, TPR-MET, resulting from a rearrangement between the translocated promoter region $(T P R)$ on chromosome 1 on the $5^{\prime}$ end, and the MET gene on chromosome 7 on the 3 ' end $(14,15)$. MET was shown to have homology with the receptor tyrosine kinase (RTK) family and to recognize the hepatocyte growth factor (HGF) as ligand (Figure 1) (16-18). MET expression is regulated at multiple layers by various mechanisms, such as DNA methylation and histone acetylation, alternative splicing, protein translation, posttranslational modifications and protein degradation $(19,20)$. The receptor encompasses several functional domains: the extracellular portion contains a region of homology to semaphorins (Sema domain), which includes the full $\alpha$ chain and the $\mathrm{N}$-terminal part of the $\beta$ chain, a plexin-semaphorin-integrin (PSI) domain and four immunoglobulin-plexin-transcription factor (IPT) domains. The intracellular part of the receptor consists of a juxtamembrane domain, a catalytic tyrosine kinase (TK) domain, and a carboxy-terminal regional multisubstrate docking site, capable of recruiting multiple downstream adaptors with Src homology-2 (SH2) domain (21). Its physiological ligand is the HGF/scatter factor (HGF/SF), a two-chain $(\alpha$ and $\beta$ ) protein produced by the activation through site-specific proteolysis of pro-HGF, a singlechain, inactive molecule (22-24). HGF/SF contains two MET-binding sites, one in the NK1 fragment, and one in the SPH domain. Ligand binding induces MET homodimerization and autophosphorylation of tyrosine residues of its catalytic domain. Other tyrosines (1,349 and $1,356)$ are then phosphorylated within the docking site and allow recruitment of a large variety of downstream effectors, including growth factor receptor-bound protein-2 (GRB2), GRB2-associated binding protein 1 (GAB1), phospholipase $\mathrm{C} \gamma 1$ (PLC $\gamma 1$ ), SRC, the p85 regulatory subunit of phosphatidylinositol-3 kinase (PI3K) and the 


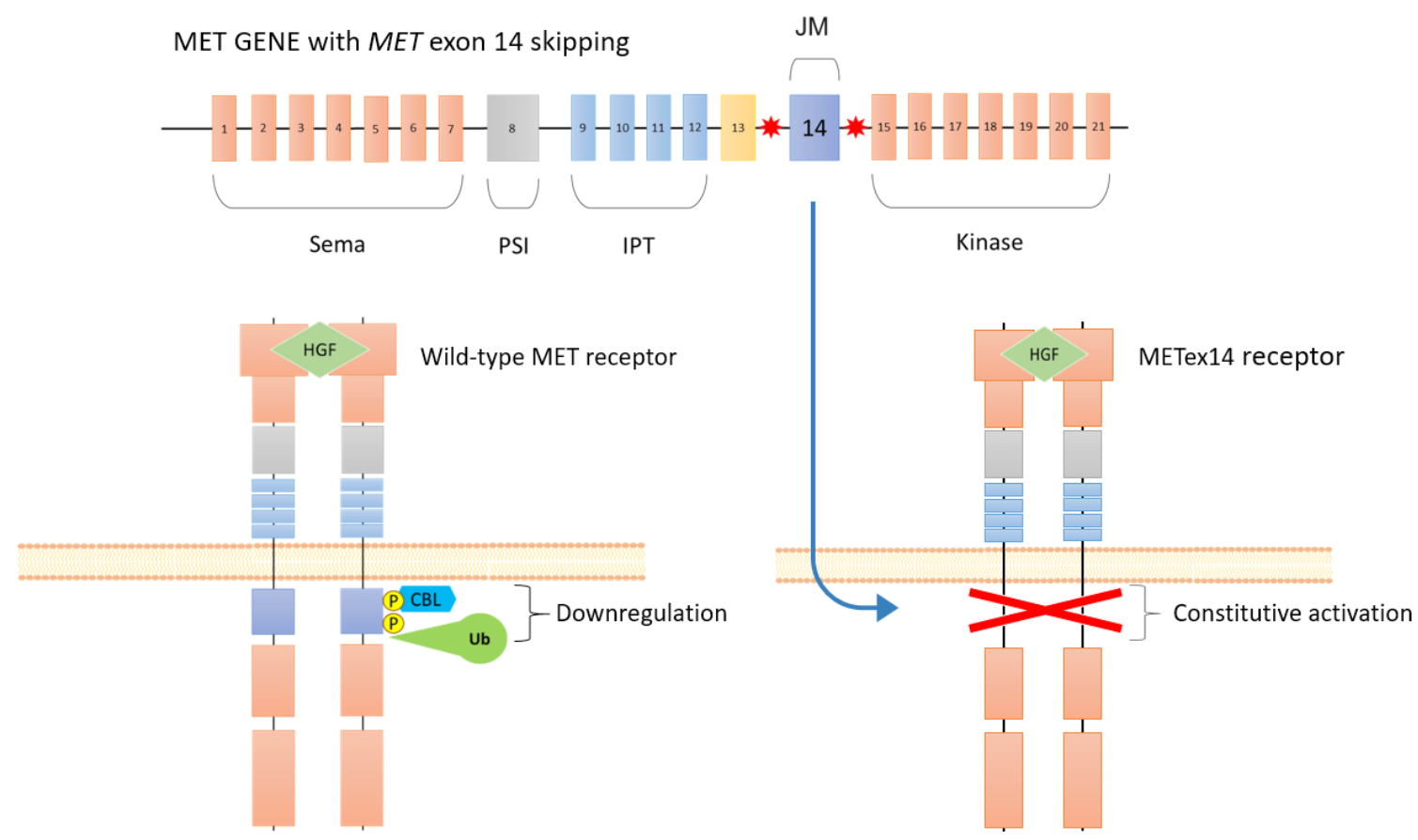

Figure 1 MET wild type and MET with exon 14 skipping mutations leading to loss of the juxtamembrane domain and constitutive activation. IPT, immunoglobulin-plexin-transcription; HGF, hepatocyte growth factor; PSI, plexin-semaphorin-integrin; JM, juxtamembrane.

signal transducer and activator of transcription 3 (STAT3) $(6,25)$. MET activates multiple signal transduction pathways, including the RAS-mitogen-activated protein kinase (MAPK) cascade, the PI3K-AKT pathway, the Signal Transducer and Activator of Transcription (STAT) and NF$\kappa \mathrm{B}$ pathway, involved in cell proliferation, differentiation, cell motility, invasion and survival. Moreover, by regulating cell-matrix adhesion and promoting cytoskeletal changes and cell migration, MET plays a key role in epithelial to mesenchymal transition (EMT) $(6,24)$. The pleiotropic biological effects of MET activation also rely on crosstalk and signaling cooperation with various surface membrane proteins (26), including the MET homologue RON, and other TKs, such as ROR1, CD44, integrins, plexin B1 and EGFR. Similar to other RTKs, MET is downregulated through a variety of mechanisms. On ligand activation, MET is internalized through clathrin-mediated endocytosis, as a mechanism to prevent sustained signaling activation. The internalized receptor is delivered to endosomal compartments and can be degraded or recycled to plasma membrane. A key protein involved in internalization and degradation is the E3 ubiquitin ligase casitas B-lineage lymphoma (CBL), that recognizes the phosphorylated Tyr1003 in the juxtamembrane domain, encoded by exon 14 of $M E T$, and recruits regulatory components of endocytic vesicles, while on the other hand, engages ubiquitin ligases to ubiquitylate MET that is then degraded in a proteasomedependent manner (27-29). Other mechanisms involved in MET downregulation have been described, including proteolysis by ADAM metalloproteases and shedding of the extracellular domain (30).

While HGF is mainly expressed and secreted by mesenchymal cells, such as fibroblasts, MET is wider expressed by epithelial cells of a variety of tissues, but also found on endothelial cells, neurons, hepatocytes, and hematopoietic cells, and has been demonstrated to be involved in a variety of physiological processes during embryonic development and in tissues repair in adults [reviewed in (24)].

\section{MET alterations driving tumorigenesis}

A deeper knowledge of the biology of this receptor in normal tissues has been helpful for understanding the roles of HGF and MET in cancer. Indeed, aberrant HGFMET signaling promotes tumor proliferation, invasive growth, angiogenesis and metastasis (6). MET expression and signaling is essential for expansion and maintenance of 
cancer stem cells (CSCc) (31).

MET constitutive activation is a common event in several cancer types and can be the result of various mechanisms, including excessive autocrine or paracrine production of HGF, MET overexpression or genetic abnormalities of MET, such as gene copy-number amplification and point mutations. Other mechanisms include inadequate internalization and degradation and receptor crosstalk $(6,32)$.

MET overexpression, due to increased gene copy number or transcriptional regulation, is commonly detected in many different solid tumors, including lung cancer, and correlates with poor prognosis (33-36). MET amplification, found in a small percentage of tumors, leads to kinase overexpression and constitutive activation of downstream survival signals and has been demonstrated to be an acquired resistance mechanism to different classes of EGFR TKIs $(37,38)$. Activating point mutations in the kinase domain occur in different sporadic and inherited cancers and data suggest a correlation with progression and metastasis. These mutations, such as D1228N, Y1235D, and M1250T, lead to constitutive MET activation and can contribute to tumor development (7). In lung cancer, oncogenic mutations can be also found in the Sema domain (exon 2), and the juxtamembrane domain (exon 14) (6,7). MET mutations located within the Sema domain could affect ligand binding rather than promoting kinase activity (6). The expression of MET variants harboring point mutations within the juxtamembrane domain, including R988C and T1010I, was correlated with enhanced tumorigenicity and metastatic potential in lung cancer (39).

However, different studies have described MET mutations identified outside the kinase domain as germline polymorphisms $(7,40-42)$ that do not induce kinase activation and may not act as oncogenic alterations. Hence, it remains crucial to fully elucidate the functional consequences of MET mutations and their relevance in oncogenesis. Dysregulation of the MET pathway can also occur through gene rearrangements, giving rise at fusion proteins encompassing the MET intracellular domain fused with a dimerization motif that potentially leads to a constitutive dimerization and activation of the receptor. The first to be identified in lung cancer was TPR-MET $(14,15)$. New MET fusion proteins have been uncovered by RNA sequencing analysis from The Cancer Genome Atlas (TCGA) data and found to occur at low frequencies in different tumor types (43). A kinesin family member 5B gene (KIF5B)-MET fusion was detected in a sample from a patient with lung adenocarcinoma (43). More recently, other MET fusion partner genes have been identified in lung cancer patients by using next-generation sequencing (NGS) technologies. These genes include StAR-related lipid transfer domain-3 $\mathrm{N}$-terminal like (STARD3NL), human leukocyte antigen $(H L A)$-DRB1, CD47 and ataxin 7-like 1 (ATXN7L1) (44-47). MET fusions are oncogenic in cellular models, suggesting may be suitable for targeted inhibition in lung cancer. Moreover, some studies have demonstrated a significant association with clinical response to crizotinib (44-46), suggesting a potential role of MET rearrangements as predictive biomarkers to MET inhibitors.

\section{MET exon 14 skipping mutations}

Somatic mutations affecting splicing sites of exon 14 of the MET gene, encoding for the juxtamembrane region, were first reported in small cell lung cancer. In this study, two tumor samples were found to harbour a two basepair insertion within the intron 13 of MET, that could potentially cause alternative mRNA splicing and skipping of exon 14.

Thereafter, MET exon 14 (METex14) splice site mutations were reported in NSCLC and other tumor types $(8,39)$. These mutations, including point mutations, insertions/deletions (indels) or large deletions, cause "exon 14 skipping" and permanent loss of the juxtamembrane region from the MET transcript (Figure 1). This region contains a tyrosine residue (Tyr 1003) that, as previously commented, is responsible for efficient recruitment of the ubiquitin ligase CBL which is required for MET internalization and degradation. Moreover, the domain includes a serine residue (S985), which is phosphorylated by protein kinase $\mathrm{C}$ (PKC) that contributes to terminating the receptor kinase activity. Hence, loss of MET exon 14 leads to increased MET stability and expression on cell surface and prolonged activation of downstream signaling pathways, thus conferring oncogenic properties to cells (Figure 1) (48-50). Consistent with this, insertion of exon 14 into the oncogenic gene fusion TPR-MET, which consists of the $M E T$ sequence downstream from the juxtamembrane domain fused to the dimerization motif of $T P R$, resulted in decreased oncogenic potential $(6,51)$.

MET exon 14 mutations in NSCLCs are found in approximately $3-4 \%$ of NSCLCs, mainly in adenocarcinoma, adenosquamous carcinoma, and the rare sarcomatoid histology, in older patients (52-57).

A meta-analysis including 12 studies comprising 18,464 NSCLCs, reported that the mutation is more likely to occur 
in females and non-smokers (58). Contrasting results have been reported from clinical studies regarding the association of smoking status with MET exon 14 mutations $(53,58,59)$. Both MET exon 14 mutations and MET amplification have been correlated with poor patient prognosis in NSCLC $(54,58)$. Different DNA- and RNA-based techniques, including RT-PCR or NGS, have been used to identify $M E T$ exon 14 skipping events in lung cancer, although with some differences between these two diagnostic approaches. For example, it is difficult to obtain optimal quality and/or quantity of RNA from clinical samples (59-61).

Of note, similar to other activated oncogenes, $M E T$ exon 14 mutations have been shown to be mutually exclusive with other known drivers, including EGFR, KRAS, BRAF mutations and $A L K$ or ROS1 rearrangements, but to cooccur with other alterations, such as MET, MDM2, CDK4 and EGFR amplification, and also with PI3KCA mutations $(8,55,58,60,62)$.

Overall, MET exon 14 mutations have emerged as attractive targetable genomic alterations in NSCLCs, thus prompting a number of clinical trials testing different classes of MET inhibitors in this subset of patients.

\section{Therapeutic agents against the HGF/MET axis}

Several therapeutic strategies have been developed to target MET signaling, including anti-HGF or anti-MET antibodies and small molecule TKIs. These agents are currently in different stages of clinical testing.

Preclinical studies suggested that MET blockade was effective only in tumors harboring MET genomic alterations, including amplification or activating mutations; whereas targeting the HGF/MET pathway in tumors with wild type $M E T$ had little or no effect on cancer cell growth and did not influence cytotoxicity of chemotherapy $(8,63)$. This preclinical evidence has been supported by negative results from some phase III randomized trials combining EGFR TKIs and MET inhibitors $(64,65)$, such as the TKI tivantinib or the anti-MET monoclonal antibody onartuzumab, including pre-treated NSCLC patients unselected for any oncogenic alterations. Indeed, in a post hoc analysis, a longer OS with tivantinib was observed in the subgroup of patients with tumors displaying high MET copy number gain (64). Also, the use of the association with erlotinib in patients with MET-expressing tumors, as assessed by immunohistochemistry, did not show any survival benefit compared to placebo (65).

In initial reports, clinical responses to MET inhibition were observed in lung cancer patients with MET exon 14 skipping mutations $(8,48,50)$.

\section{Multitarget TKIs}

MET TKIs are small molecules that can generally be divided into three groups, according to their structure and mode of binding with MET $(24,66)$. Type I and II are both ATP-competitive inhibitors and are those mostly being evaluated in clinical trials. Type I inhibitors occupy the ATP-binding pocket and bind to the activation loop (A-loop), with Y1230, to block catalytic activation. Type Ia, such as crizotinib, interacts with the solvent front residue G1163, while type Ib inhibitors, such as capmatinib, tepotinib, savolitinib, and APL-101, are independent of G1163 interaction. Type II inhibitors, such as cabozantinib, foretinib, merestinib and glesatinib, also occupy the ATPbinding pocket and extend to the deep hydrophobic back pocket and bind to the inactive "DFG out" conformation of MET. Hence, binding is independent of interactions with the A-loop. Type III are non-ATP competitive allosteric inhibitors binding outside the ATP pocket.

\section{Crizotinib}

Crizotinib is an oral small-molecule, multikinase inhibitor of ALK, ROS1, RON and MET and is currently approved for treatment of advanced NSCLC patients harboring $A L K$ or ROS1 rearrangements (67-69). Crizotinib acts as a type Ia MET TKI and competes for the ATP-binding site of the RTK, thereby preventing activation of downstream signaling pathways. Treatment with crizotinib of tumor cell lines with $M E T$ exon 14 skipping mutations impaired their viability and inhibited downstream pathways activation (50). A number of clinical reports supported this preclinical evidence by demonstrating that the use of crizotinib induced objective responses in patients with advanced NSCLC with $M E T$ exon 14 alterations $(50,55,70-74)$. The antitumor activity of crizotinib in advanced, $M E T$-exon 14-altered NSCLC patients was prospectively assessed in an expansion cohort of the multicenter phase 1 study, PROFILE 1001 (Table 1) $(75,76)$. Overall, 69 patients with advanced NSCLC harboring MET exon 14 alterations, as assessed mostly by using DNA- or RNA-based, were included in the study. The median age was 72 years old. Most patients had adenocarcinoma and were former smokers and the majority of these had received $\geq 1$ previous lines of treatment for advanced disease. Among the 65 patients evaluable for tumor response, the objective response rate (ORR) was 


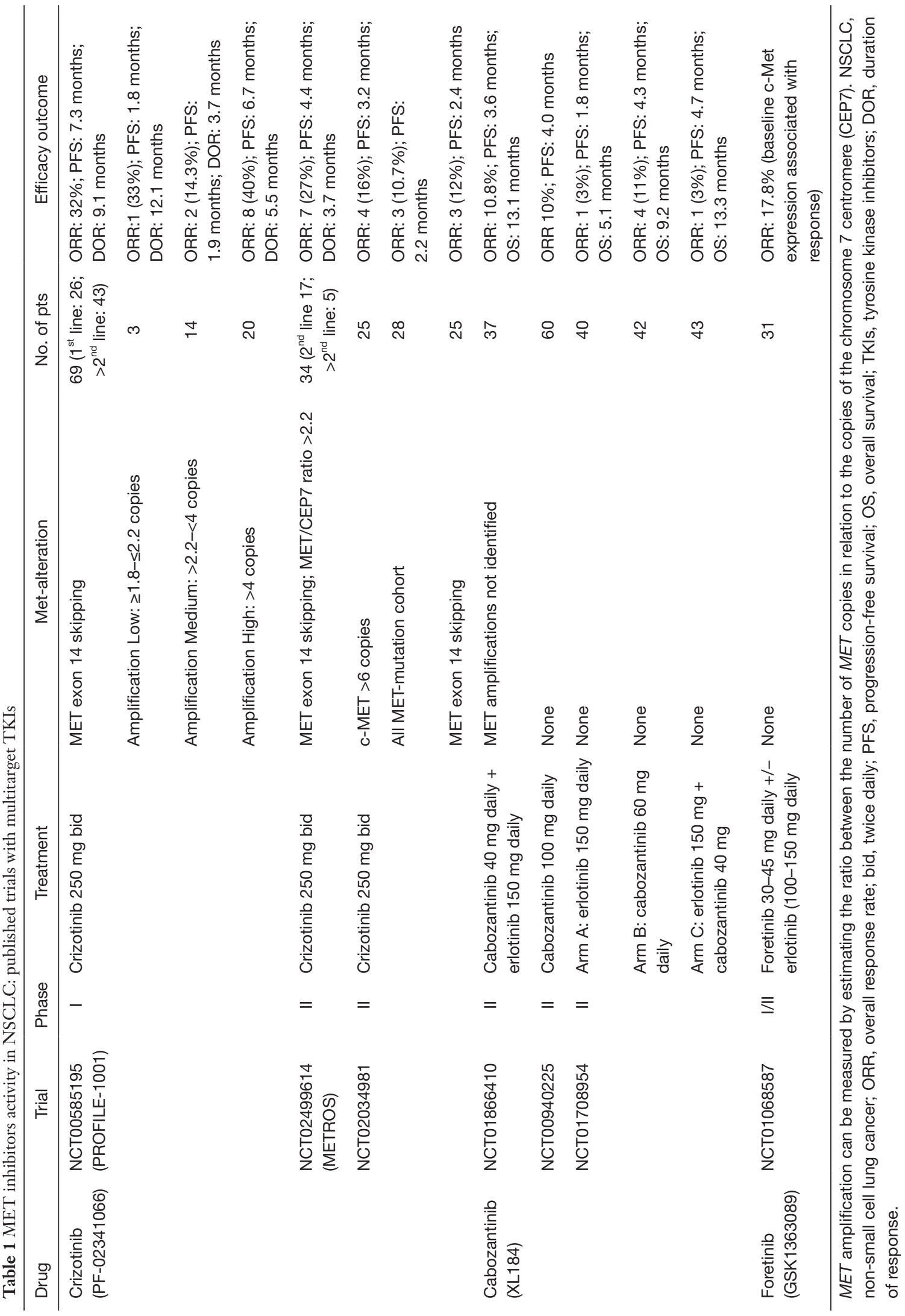


$32 \%$. Responses were rapid, durable (57\% of patients having a response of $\geq 6$ months) and were observed independent of the type of MET alterations (76). Interestingly, no significant difference in responses was observed between ctDNA-positive versus ctDNA-negative patients. The median PFS was 7.3 months and the probability of survival at 6 and 12 months was $87 \%$ and $70 \%$, respectively (76). By local testing, increased concurrent MET copy number was observed in 2 patients, achieving durable disease control of more than 6 months. A significant association between additional concurrent genomic alterations in TP53, MDM2 and $C D K N 2 A$ and clinical outcomes was not found. The overall safety profile of crizotinib was consistent with that observed in previous studies, with edema, vision disorders, nausea, diarrhea and vomiting being the most common treatment-related adverse events (TRAEs), most of grade 1 and 2. Grade 3 included elevated transaminases and dyspnea. Permanent discontinuation for TRAEs occurred in $7 \%$ of patients. This study definitively underscores the importance of testing for MET exon 14 alterations in the clinic with sensitive, NGS techniques in tissue specimens, as well as in liquid biopsy. Based on initial data from this study, crizotinib as a MET inhibitor received breakthrough therapy designation from the United States Food and Drug Administration (US FDA) in 2018 for previously treated metastatic NSCLC in patients with MET exon 14 skipping. Other prospective phase II studies have evaluated crizotinib in this molecularly-defined subgroup of patients (77,78). Limited clinical benefit with crizotinib in METderegulated NSCLC patients was observed in the phase II METROS study. In the cohort including patients with both MET amplification and exon 14 mutations, ORR was 27\%, median PFS 4.4 months, and OS 5.4 months, with no significant differences between $M E T$-amplified and exon 14-mutated patients (Table 1) (78).

The above data, and results from a recent metaanalysis (79), demonstrate of crizotinib in this oncogeneaddicted NSCLC subgroup is lower compared to results in ROS1 or $A L K$-rearranged tumors, or to the efficacy of other oncogene-directed therapies, suggesting type Ia inhibitors are not the optimal agents for this subgroup of patients. Indeed, other more selective MET TKIs have been tested in clinical trials.

\section{Cabozantinib}

Cabozantinib (XL-184) is a small molecule, nonselective inhibitor of MET that targets multiple kinases, including VEGFR1-3, RET, TIE2, FLT-3 and KIT, currently approved for treatment of medullary thyroid carcinoma, renal cell carcinoma and hepatocellular carcinoma $(80,81)$. Cabozantinib showed preclinical anti-tumor activity in a variety of mouse models, including lung cancer (82). In unselected, pretreated NSCLC patients, positive results in terms of improved PFS emerged from a randomized phase II study of cabozantinib with erlotinib or cabozantinib alone, compared to erlotinib, with acceptable tolerability (Table 1) (83). This combination was also tested in EGFR mutant NSCLC patients who progressed on prior EGFR TKI. This phase II study enrolled 37 patients, including $15(40.5 \%)$ patients who had received more than 2 prior lines of systemic therapy. Overall, 4 (10.8\%) patients had partial responses and the majority of patients $(59.5 \%)$ had stable disease, with PFS and OS of 3.6 months and 13.3 months, respectively (Table 1) (84). Of note, activity was not correlated to the type of EGFR mutations or presence of T790M, and none of the patients with available tissue had evidence of MET amplification at baseline (84). Its role in patients with MET alterations has not been yet defined. In the study by Paik et al, cabozantinib induced a complete metabolic response as third line therapy in a patient with MET exon 14 alteration (50). Subsequent reports demonstrated systemic and intracranial activity of cabozantinib in this subset of patients $(85,86)$. The agent is currently being tested in phase II clinical trials in patients with NSCLC and MET amplification or MET exon 14 skipping mutations (CABinMET study, NCT03911193) and in NSCLC with RET rearrangements or other oncogenic alterations, including ROS1 or NTRK rearrangements and $M E T$ or $A X L$ alterations, including amplification, mutations or protein overexpression (NCT01639508) (Table 2).

\section{Merestinib}

Merestinib (LY2801653) is an orally bioavailable, ATPcompetitive, multitargeted inhibitor of MET and of other TKs involved in tumor cell proliferation, growth and angiogenesis, including AXL, ROS1, VEGFR2, FLT3, DDR1-2, MERTK and NTRK1-3, that showed tolerable safety profile and potential anticancer activity in pretreated, advanced cancers $(87,88)$. In preclinical in vitro and in vivo NSCLC models, this agent displayed tumor growth inhibition in tumor cell lines and patient-derived tumor xenograft models as a single agent or when used in combination with other antineoplastic drugs. In a metastatic lung orthotopic tumor model, LY2801653 treatment significantly inhibited both primary tumor growth and metastasis (89). Treatment with merestinib decreased 
Table 2 Ongoing trials with MET Inhibitors in NSCLC

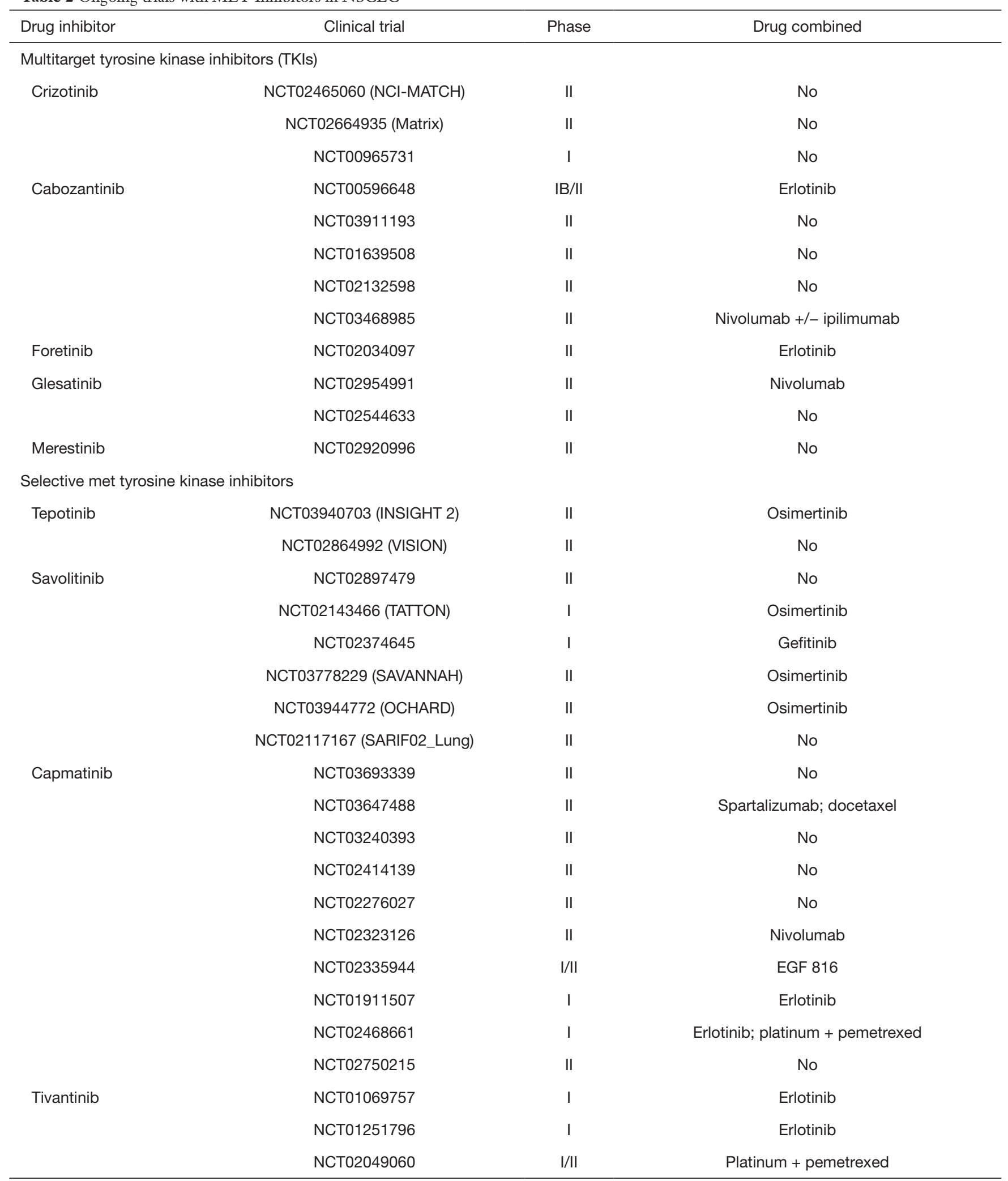

Table 2 (continued) 
Table 2 (continued)

\begin{tabular}{|c|c|c|c|}
\hline Drug inhibitor & Clinical trial & Phase & Drug combined \\
\hline \multicolumn{4}{|c|}{ Anti-met antibodies } \\
\hline \multirow[t]{4}{*}{ Onartuzumab } & NCT01887886 & III & Erlotinib \\
\hline & NCT01519804 & II & Platinum + paclitaxel \\
\hline & NCT02031744 & III & Erlotinib \\
\hline & NCT02044601 (BATTLE-XRT) & $\mathrm{I} / \mathrm{II}$ & Erlotinib \\
\hline Telisotuzumab & NCT03574753 (Lung-MAP S1400K) & II & No \\
\hline JNJ-61186372 & NCT02609776 & 1 & No \\
\hline Ficlatuzumab & NCT02318368 & II & Erlotinib \\
\hline
\end{tabular}

NSCLC, non-small cell lung cancer.

phosphorylation of key downstream proteins, including CBL, PI3K and STAT3, and resulted in inhibition of tumor growth by inhibiting proliferation and angiogenesis in mouse xenograft models of lung cancer (90). Merestinib showed activity as single agent or when combined with the MET-targeting antibody, emibetuzumab, in a gastric cell line and xenograft model bearing MET exon 14 mutations (91). A phase II trial of merestinib in NSCLC with $M E T$ exon 14 skipping mutations or advanced solid cancers harboring NTRK1-3 rearrangements is ongoing (NCT02920996) (Table 2).

\section{Glesatinib}

Glesatinib is an oral, ATP-competitive TKI with activity against MET, VEGFR1-3, AXL, RON and TIE2. Glesatinib is a unique type II MET inhibitor, and binds to MET independently of interactions with the A-loop, suggesting its activity against MET mutations involving the residues D1228 and Y1230 that confer resistance to type I MET inhibitors. Glesatinib demonstrated activity in tumor cell lines in vitro and marked regression of cell- and patientderived tumor models harboring METex14 del mutations in vivo. A durable clinical response was observed in a NSCLC patient with $M E T$ exon 14 mutation receiving this agent (92). Glesatinib also demonstrated preclinical and clinical activity against secondary mutations within the MET activation loop conferring resistance to type I inhibitors (92).

A phase II trial of glesatinib in advanced, pre-treated,
NSCLC patients with activating genetic MET alterations (mutation or amplification), as assessed in tumor tissue or ctDNA (NCT02544633), has been completed and results are awaited (93). Another ongoing trial is evaluating this agent with the anti-PD1 nivolumab in advanced NSCLC (NCT02954991) (Table 2). High mutant allele frequency of METex14 was shown to predict tumor response to glesatinib in phase 1 and 2 studies (94).

\section{Selective MET TKIs}

\section{Capmatinib}

Capmatinib (INC280) is an oral, ATP-competitive, inhibitor of MET. Capmatinib demonstrated to be potent and highly specific against MET, with more than 10,000fold selectivity over other human kinases, and to inhibit MET-dependent tumor cell proliferation and tumor growth in MET-driven mouse tumor models (95). Significant antitumor activity was observed in cell line- or patientderived xenograft models with various mechanisms of MET activation, including $M E T$ exon 14 alterations (96). Of note, in those models where MET activation co-occurred with other oncogenic alterations, such as EGFR mutations, the combination of capmatinib and EGFR TKIs resulted in enhanced anticancer activity (96). In the initial report by Frampton et al., two NSCLC patients with MET exon 14 mutations showed partial responses to capmatinib (8). These patients were included in a phase I open-label, dose- 
escalation study with an expansion part (NCT01324479) to assess the safety and tolerability of capmatinib, at the recommended dose of $400 \mathrm{mg}$ (tablets) or $600 \mathrm{mg}$ (capsules) twice daily, in patients with advanced MET-dysregulated NSCLC (97). Clinically meaningful antitumor activity was observed in patients with MET GCN 6 and/or METex14 mutations (Table 3). Tumor responses were observed in three out of the four patients with MET exon 14 mutations, including one confirmed complete response. Importantly, in this phase I trial capmatinib had an acceptable safety profile. The most frequent drug-related grade 3 or 4 AEs, were nausea, peripheral edema and fatigue, occurring in $4 \%$ of patients (97). The phase II GEOMETRY mono-1 (NCT02414139) was a multicenter, non-randomized, openlabel, multi-cohort, phase 2 study evaluating capmatinib (400 mg orally twice daily) in EGFR wild-type and $A L K$ negative NSCLC patients with $M E T$ alterations, including MET exon 14 skipping mutation or MET amplification. Patients were assigned to cohorts on the basis of MET status and previous lines of therapy. Primary endpoint was ORR assessed by a blinded independent review committee (BIRC) and secondary objectives included duration of response (DoR), PFS, OS and safety. Preliminary results from cohort 4 and $5 \mathrm{~b}$, including patients with MET exon 14 mutations (regardless of MET amplification status/gene copy number), pre-treated with systemic therapy (not with a prior MET inhibitor) or treatment-naïve, respectively, showed that the ORR and PFS with capmatinib were higher in treatment-naïve patients compared to pre-treated patients (98). Rapid and durable responses were observed across both cohorts. Capmatinib also showed significant intracranial activity and had a favorable safety profile, with the majority of the AEs being of grade 1/2. The most common treatment-related AEs were peripheral edema, nausea, increased blood creatinine and vomiting (98). On May 6, 2020, based on these positive results, the FDA granted accelerated approval to capmatinib (TABRECTA, Novartis) for adult patients with metastatic NSCLC whose tumors have a MET exon 14 skipping mutation and also approved the FoundationOne CDx assay (Foundation Medicine, Inc.) as a companion diagnostic for capmatinib. Recently, final results from the GEOMETRY mono-1 study have been published and showed robust antitumor activity of the agent in this molecularly-defined subgroup of NSCLC. Among NSCLC patients with MET exon 14 skipping mutations, ORR was $41 \%$ and DoR 9.7 months in pre-treated patients. In treatment-naïve patients, ORR and DoR were $68 \%$ and 12.6 months (Table 3). Also, median PFS was improved in patients who had not received previous treatment (12.4 months) than in previously treated patients (5.4 months). A total of 12 out of the 13 patients evaluable by the independent neuroradiologic review committee had intracranial disease control, including 7 with an intracranial response. No significant differences in response to capmatinib according to the type of genetic alteration causing MET exon 14 skipping mutations or the co-occurrence of MET amplification were observed (99). In this study, capmatinib showed evidence of activity also in patients with $M E T$ amplification and GCN $\geq 10$ NSCLC, although at less extent than in patients with MET exon 14 alterations. The ORR was $29 \%$ in pre-treated patients and $40 \%$ in not previously treated. Of note, most patients included in these cohorts of the study were male and had a history of smoking. The favorable safety profile was confirmed in these final results, showing mainly grade $1 / 2$ and reversible adverse events; the most frequent of these were peripheral edema, nausea, vomiting and increased blood creatinine level (99).

Expansion cohort 7, including patients with NSCLC with a $M E T$ exon 14 skipping mutation who had not received treatment previously, is ongoing.

Additional Phase II trials (NCT03693339, NCT02750215) of capmatinib in patients with MET exon 14 mutationpositive NSCLC are ongoing, including a study of capmatinib with spartalizumab (an anti-PD-1 antibody) compared with capmatinib alone in treatment-naïve NSCLC (NCT04323436) (Table 2).

\section{Tepotinib}

Tepotinib is an oral, ATP-competitive MET inhibitor (type Ib). Screening against $>400$ kinases showed high selectivity of tepotinib for MET. In vivo, tepotinib was associated with tumor regression in murine xenograft models of human cancers, regardless of whether or not MET activation was dependent on HGF (100). Moreover, tepotinib showed promising clinical activity in patients with MET-driven tumors (101). The phase II VISION study (NCT02864992), evaluated the efficacy and safety of tepotinib in advanced NSCLC patients with $M E T$ alterations, including $M E T$ exon 14 skipping mutations and MET amplification. Results from patients with $M E T$ exon 14 skipping mutations, as detected on NGS in tissue- or liquid-biopsy, have been recently published (Table 3) (102). A total of 152 patients received tepotinib, including 11 patients with baseline brain metastases. Most of the enrolled patients were adenocarcinoma, median age was 74 years and 56 had 


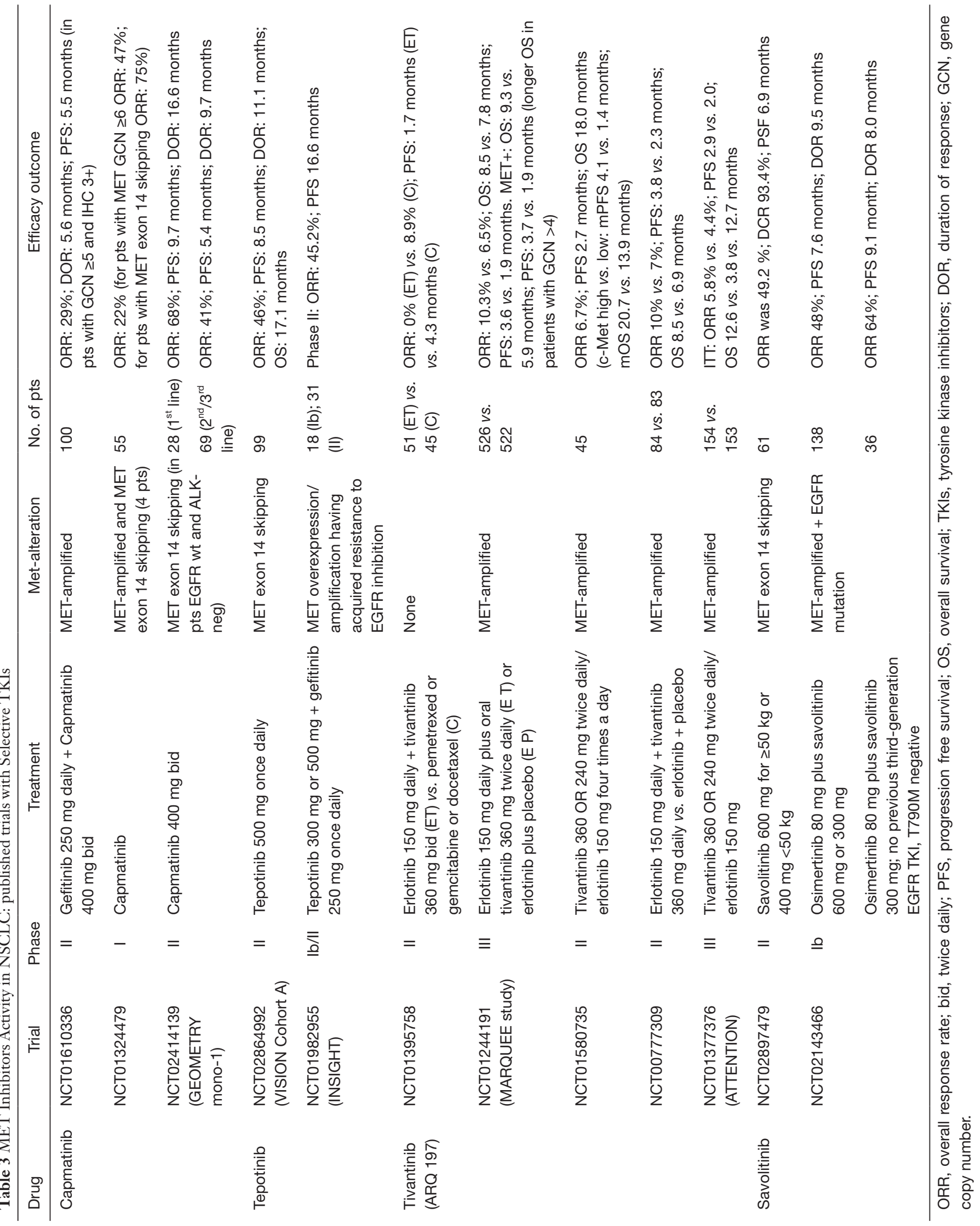


received previous therapies, including immunotherapy. In the combined-biopsy group, the response rate by independent review was $46 \%$, all were partial responses. Responses were rapid and durable (median DoR of 11.1 months). The median duration of PFS was 8.5 months in the combinedbiopsy group and median duration of OS 17.1 months, according to not mature data. Clinical outcomes were similar in the two biopsy categories. Of the 51 patients who had matched baseline and on-treatment biomarker profiles from liquid biopsies, 34 (67\%) had a molecular cfDNA response, of whom $24(71 \%)$ showing radiologic response according to independent review, suggesting high concordance between molecular and clinical response. TRAEs of grade 3 or higher were reported in $28 \%$ of the patients, with peripheral edema being the main toxic effect, observed in $7 \%$ of patients. Overall, the patients' quality of life was maintained during receipt of tepotinib (102). On September 2019 , the drug was granted breakthrough therapy status by the FDA for advanced NSCLC harboring MET exon 14 skipping alterations and, in March 2020, was first approved in Japan for the treatment of this molecularly-defined subgroup of NSCLC patients, together with its companion diagnostic assay (ArcherMET CDx) (103). Tepotinib has also demonstrated activity in the setting of acquired resistance to EGFR TKIs. Results from the randomized, phase II part of the INSIGHT trial in patients with EGFR-mutant NSCLC T790M-negative, with MET overexpression and/ or MET amplification, and resistance to prior EGFR TKI, have been reported $(104,105)$. Tepotinib in combination with gefitinib showed an ORR of $45.2 \%$ versus $33.3 \%$ for the chemotherapy groups (Table 3). Salient findings were found in patients with $M E T$ amplification where ORR was 66.7 with tepotinib plus gefitinib and $42.9 \%$ for chemotherapy. In the combination group of tepotinib plus gefitinib, median PFS was 16.6 months versus 4.2 months in the chemotherapy group, and median OS was 37.3 versus 13.1 months (104). Progression-free survival and overall survival were longer with tepotinib plus gefitinib than with chemotherapy in patients with high [immunohistochemistry (IHC)3+] MET protein expression. MET amplification correlates with the degree of MET expression in most cases. Importantly, tepotinib plus gefitinib was generally well tolerated (104). The trial highlights the need for tailored therapy, and that $M E T$ amplification could be a feasible predictive biomarker in this subset of patients, as the combination of tepotinib and gefitinib was ineffectual in patients with low MET expression (IHC2+ or less) (105). The INSIGHT 2 study (NCT03940703) is ongoing and will explore the benefit of tepotinib plus osimertinib in patients with EGFR-mutant NSCLC who have progressed on previous EGFR TKIs due to MET amplification (Table 2).

\section{Savolitinib}

Savolitinib is an oral, ATP-competitive kinase inhibitor, with high activity and selectivity for MET (106). Savolitinib has been tested in different MET-driven tumors, including gastric, papillary renal cell carcinoma and NSCLC. In the multi-arm, phase $1 \mathrm{~b}$ TATTON trial conducted in patients with advanced EGFR-mutant NSCLC and disease progression on a prior EGFR-TKI, osimertinib in combination with savolitinib was feasible and tolerable at the dose levels identified, and was associated with an ORR of $44 \%$ among the 18 patients enrolled (107) (Table 3). Of note, MET testing identified 9 patients with $M E T$ amplification by FISH. Among the 3 patients with MET amplification included in the savolitinib arm, 2 had a response. Osimertinib plus savolitinib is being studied further in patients with MET-driven resistance to EGFRTKIs (SAVANNAH; NCT03778229 and ORCHARD; NCT03944772) (Table 2).

Savolitinib has also demonstrated activity against MET exon 14 mutations in in vitro and in vivo studies, as well as in the clinical setting (108-110). A multicenter, multicohort phase II study (NCT02897479) has been conducted to evaluate the efficacy and safety of savolitinib in unresectable or metastatic pulmonary sarcomatoid carcinoma (PSC) and other NSCLC with MET exon 14 mutations (110). In the cohort of MET-inhibitor naïve patients ORR by IRC assessment was $49.2 \%$, all were partial responses (Table 3). Activity was observed both in PSC and other NSCLC groups. Disease control rate was $93.4 \%$ and DoR 9.6 months Survival data were not mature. Median PFS was 6.9 months; specifically, it was 5.6 months for treatmentnaïve group, including nearly half of patients with PSC and 13.8 months for previously treated patients. Tolerability was acceptable, with most TRAEs being edema, nausea, vomiting, increased AST/ALT and hypoalbuminemia. The incidence of $\geq$ grade 3 TRAEs was $41.4 \%$ and TRAEs leading to treatment discontinuation occurred in $14.3 \%$ patients, among which liver injury and hypersensitivity were most common (110).

\section{Other therapeutic agents targeting MET exon 14 mutations}

\section{Sym015}

Sym015 is a mixture of two recombinant humanized 
antibodies triggering non-overlapping epitopes in the extracellular domain of MET that blocks HGFMET interaction, induces receptor internalization and degradation and stimulates CDC and ADCC in vitro and in vivo, thereby improving specificity compared to TKIs. An additional advantage of this molecule is that may circumvent intracellular acquired resistance mechanisms, such as secondary kinase mutations, to MET TKIs. Preliminary clinical activity of Sym015 from an expansion cohort of the phase 2 trial (NCT02648724) in advanced NSCLC patients with $M E T$ amplification/exon 14 deletion was recently reported (111). Among the 20 patients included, 5 had partial responses (ORR 25\%) and the DCR was $80 \%$. All responses were observed in the group of MET TKI-naïve patients. For patients who were prior MET TKI-treated, DCR was $60 \%$. Median PFS for MET TKI-naïve and MET TKI pre-treated NSCLC patients was 7.4 and 5.4 months, respectively. In patients with $M E T$ exon 14 mutations TKInaïve, PFS was 9.2 months. Median OS was 9.1 months for those with MET exon 14 mutations MET TKI-treated and not reached for overall and other subgroups. Of note, ctDNA was highly concordant with tumor for MET exon 14 mutations detection. Safety was assessed in 45 patients included in all cohorts of the phase 2 study. Overall, the drug was well tolerated, with $13.3 \%$ of grade 3 TRAEs, including anasarca and hypoalbuminemia, colitis and septic shock, amylase increase, hypophosphatemia and in NSCLC patients, peripheral edema and elevated liver function tests (111).

\section{Immunotherapy in MET exon 14-mutant NSCLC}

Limited clinical benefit from anti-PD1/PD-L1 directed ICIs has been reported in NSCLC patients with MET alterations (112-114). In a retrospective analysis evaluating ICIs activity as monotherapy in advanced NSCLC with oncogenic drivers, 36 patients with $M E T$ alterations (MET amplification $\mathrm{n}=13$ or $M E T$ exon 14 mutation $\mathrm{n}=23$ ) were included. Among these patients, an ORR of $16 \%$ was observed, with median PFS and OS of 3.4 and 18.4 months, respectively (112). In another study including a larger number of patients, exon 14 mutations were mainly associated with high ( $\geq 50 \%)$ PD-L1 expression; however, the ORR with ICIs was $17 \%$ and median PFS 1.9 months (113). In this study, tumor mutational burden (TMB) was also assessed in each oncogenic subgroups. TMB is defined as the number of mutations per DNA megabase. High TMB leads to the creation of neoantigens and increases tumor immunogenicity, which can improve the response to cancer immunotherapy. TMB in MET exon 14 was low compared to wild type NSCLC, as observed in other reports $(62,98,113,115)$.

In a comprehensive genomic analysis on 60,495 NSCLC patients, MET exon 14 mutations, including base substitution, indels, deletions, and complex events spanning multiple functional sites of $M E T$, were found in $1,387(2.3 \%)$ of samples (62). TMB was significantly lower in $M E T$ exon 14-altered, compared to MET exon 14 wild type tumors. Moreover, MET exon-14-mutant NSCLC was enriched for high ( $\geq 50 \%)$ PD-L1 expression: $48 \%$ compared to $29 \%$ in WT NSCLC. No difference in PD-L1 positivity was observed across different MET exon 14 alterations (62). In contrast to previous studies, a recent study reported remarkable and durable responses to ICIs in advanced MET-mutant NSCLC patients (115). Among 13 patients treated with ICIs, $6(46.2 \%)$ had objective responses (4 partial and 2 complete), and response was maintained for 18-49 months. Despite these promising data, further data is needed to define the role of ICIs in this distinct subset of oncogene-addicted NSCLC (115).

\section{Intrinsic and acquired resistance to MET inhibitors}

Similar to other oncogene-directed therapies, treatment with MET TKIs can lead to acquisition of resistance by cancer cells through different mechanisms, including ontarget mechanisms, such as secondary mutations within the kinase domain, and off-target mechanisms, such as activation of alternative oncogenic signaling pathways (116). In MET exon 14-altered NSCLC, secondary mutations in the kinase domain of $M E T$ have been identified, mainly in single cases, and associated with resistance to different types of MET inhibitors (92,117-125). Mutations involving the A-loop residues D1228 and Y1230 confer resistance to type I MET inhibitors by altering their binding to the kinase domain, but do not affect type II inhibitors binding, that have been shown to be effective in the presence of these mutations $(92,117)$. In a series of lung cancer patients with $M E T$ exon 14 mutations receiving crizotinib, at time of progression, 9 had paired pre-/post-treatment samples to be analyzed for resistance mechanisms. Among these patients, 2 (22\%) developed on-target mechanisms, including MET D1228N and HGF amplification and 5 (44\%) were found to have potential off-target drug resistance mechanisms (123). MET exon $19 \mathrm{D} 1246 \mathrm{~N}$ mutation has also been found in a patient receiving crizotinib rather than cabozantinib (124). 
The solvent front G1163R mutation, analogous to the $A L K$ G1202R and ROS1 G2023R, confers resistance to type Ia TKI, such as crizotinib, that mainly binds to this residue, but not to type Ib or II TKIs $(92,125)$. Fujino et al. analyzed secondary mutations as resistance mechanisms to different type I, II and III MET TKIs in cells lines expressing MET exon 14 alterations. In this study, D1228 and Y1230 were common resistance mutations for type I TKIs, whereas the L1195 and F1200 for type II TKIs. The L1195V alters the C-terminus of the alpha-helix, and also confers resistance to crizotinib $(92,125)$. In MET TKI-resistant tumors, alternative signaling pathways can be activated, so called bypass tracks, through different RTKs or downstream molecules activation. These off-target mechanisms include activation of crucial intracellular pathways, such as the MAPK pathway and the PI3K/AKT pathway activation. Alterations in key genes activating the MAPK pathway, such as KRAS amplification or KRAS mutations, have been described (123,126-128). Acquired gene amplification of EGFR, HER2, HER3, BRAF and MDM2 have also been reported in resistant tumor samples $(123,128)$.

Genomic alterations occurring at time of acquired resistance to MET TKIs were evaluated by using plasma and tissue NGS in 20 patients. On-target mechanisms of resistance were identified in $35 \%$ of patients and included MET mutations (H1094, G1163, L1195, D1228, Y1230), and high levels of amplification of MET exon 14-mutant allele, whereas off-target mechanisms were found in $45 \%$ of patients and included $K R A S$ mutations and amplification of KRAS, EGFR, HER2, BRAF (128). Multiple MET kinase mutations were detected in some cases. The H1094Y mutation was detected in tissue after treatment with glesatinib (128). Although its impact in mediating resistance to TKI is still unknown, it seemed to reduce the ability of glesatinib to dephosphorylate MET and to be particularly sensitive to the type I MET TKI savolitinib. Two patients with on-target resistance, showed objective response when switching to a structurally different MET TKI, suggesting that the optimal therapeutic sequencing should be defined based on underlying mechanisms of resistance. Of note, activation of PIM kinases promotes resistance to MET inhibitors in NSCLC and gastric cancer cells with MET amplification and could have a role also in NSCLC with exon 14 mutations (129).

Phosphoinositide 3-kinases (PI3K) pathway alterations may also confer primary resistance to MET TKIs. In a recent work, tumor samples from patients with MET exon 14 alterations were analyzed by using targeted NGS and immunohistochemistry. PI3KCA mutations were found in 2 of $65(3 \%)$ samples and loss of PTEN in 6 of 26 (23\%) samples (130). Patients with these alterations did not respond to MET TKI therapy. Interestingly, PI3K inhibition restored sensitivity to MET TKIs in METex14mutated cell lines with a PI3K pathway alteration, thus proposing the preclinical rationale for a combined therapeutic strategy (130). Co-occurring RAS-MAPK pathway gene alterations in lung cancer patients with MET exon 14 mutations have been shown to correlate with decreased response to MET TKI treatment. In METex14mutant expressing cells, resistance to MET TKI associated with KRAS overexpression or NF1 downregulation, was reversed with the combination of crizotinib and the MEK inhibitor trametinib. These data suggest to perform deep genomic analysis of tumor samples to find out co-occurring alterations that can potentially respond to front-line approaches with combination therapies (e.g., MET TKI and MEK inhibitor) to enhance clinical outcomes (131). The study by Rotow et al. (131), was performed on ctDNA by targeted NGS. The use of liquid biopsy is crucial for identification of resistance mechanisms. Liquid biopsy has the possibility to capture tumor heterogeneity and provide a wider representation of the entire genomic landscape, including primary tumor and multiple metastatic sites. In addition, the feasibility to collect multiple specimens at various time points, before and during treatment, can be exploited to track the emergence of heterogenous mechanisms of resistance.

Most mechanisms of primary and acquired resistance to MET TKI in MET exon 14 mutant-NSCLC should further be elucidated. In this context, the role of other potential biomarkers, such as Y-box binding protein (YB-1) should be further analyzed (105). YB-1 is activated via AKT or ERK, or both, and has been shown to regulate the metastasis associated with the colon cancer-1 (MACC-1)MET pathway in lung adenocarcinoma (132). The yesassociated protein (YAP1)-FOXM1 pathway has recently been associated with resistance to EGFR inhibitors (133), highlighting potential similarities among the mechanisms of resistance to different TKIs. A potential role might be played by the ATP-binding cassette transporters that have been correlated to resistance to chemotherapeutic agents and to ALK TKIs. In MET-amplified cells resistant to crizotinib, overexpression of ATP-binding cassette subfamily B member 1 (ABCB1) was associated with CSClike properties and phenotype. Inhibition of $A B C B 1$ reversed resistance to MET inhibitors (134). 


\section{Conclusions and future perspectives}

Oncogene-directed therapies have markedly improved the management and prognosis of NSCLC patients over the last years. MET has been demonstrated to be a worthy therapeutic target in NSCLC due to its multiple roles in cancer development and progression, as well as in the acquired resistance to targeted agents, such as EGFR TKIs. A number of clinical trials are currently investigating HGF/MET inhibitors, as single agents or in combination with other agents, in different settings of disease (Table 2). However, a number of studies with potential effective MET inhibitors conducted in unselected NSCLC patient populations have reported negative results, suggesting the need of better selection of patients based on specific MET alterations.

$M E T$ exon 14 mutations have been identified as key oncogenic drivers in NSCLC, conferring sensitivity to MET TKIs in preclinical and clinical studies. The use of a MET TKI, including crizotinib, capmatinib and glesatinib, at any line of treatment in NSCLC patients with MET exon 14 mutations is associated with longer overall survival (135).

Significant clinical activity has been prospectively demonstrated by more selective MET TKIs in METmutant NSCLC patients, with recent approval of capmatinib by FDA for this subgroup of patients. This evidence underscores the importance of routine testing NSCLC tumor samples for MET alterations.

Despite promising results, many questions remain unanswered. Indeed, the optimal sequencing of MET TKIs remains to be determined, since the acquisition of secondary resistance mutations can impair the activity of some TKIs and enhance that of others, depending on their binding modalities. Moreover, the presence of co-occurring alterations can also confer primary resistance to MET blockade, thus likely explaining the lower responses of these agents compared to those observed with other TKIs, such as EGFR- or ALK-TKIs. A deeper understanding of the molecular background associated with MET exon 14 mutations may suggest novel rational combinatorial or sequential therapeutic strategies to improve clinical outcomes. The use of liquid biopsy, in addition to tissue biopsy, for genomic analyses can help the identification of MET exon 14 skipping mutations and may help track the emergence of resistant subclones over the course of treatment.

The role of MET gene or protein alterations in the development of resistance to EGFR TKIs in EGFR- mutant NSCLC has been well established and results from trials with combination of EGFR and MET TKIs are encouraging (Table 2). Other combination strategies, including MET TKIs and ICIs, need to be further explored. Finally, although MET gene fusions are relatively rare, some data suggest they could represent promising predictive biomarkers for targeted therapies in lung cancer to be screened at baseline together with other more common driver alterations.

\section{Acknowledgments}

Funding: None.

\section{Footnote}

Reporting Checklist: The authors have completed the Narrative Review reporting checklist. Available at http:// dx.doi.org/10.21037/tlcr-20-1113

Conflicts of Interest: The authors have completed the ICMJE uniform disclosure form (available at http://dx.doi. org/10.21037/tlcr-20-1113). RR serves as an unpaid Editorin-Chief of Translational Lung Cancer Research from June 2019 to May 2022. The other authors have no conflicts of interest to declare.

Ethical Statement: The authors are accountable for all aspects of the work in ensuring that questions related to the accuracy or integrity of any part of the work are appropriately investigated and resolved.

Open Access Statement: This is an Open Access article distributed in accordance with the Creative Commons Attribution-NonCommercial-NoDerivs 4.0 International License (CC BY-NC-ND 4.0), which permits the noncommercial replication and distribution of the article with the strict proviso that no changes or edits are made and the original work is properly cited (including links to both the formal publication through the relevant DOI and the license). See: https://creativecommons.org/licenses/by-nc-nd/4.0/.

\section{References}

1. Planchard D, Popat S, Kerr K, et al. Metastatic non-small cell lung cancer: ESMO Clinical Practice Guidelines for diagnosis, treatment and follow-up. Ann Oncol 2018;29:iv192-iv237. 
2. Lindeman NI, Cagle PT, Aisner DL, et al. Updated molecular testing guideline for the selection of lung cancer patients for treatment with targeted tyrosine kinase inhibitors: guideline from the college of American pathologists, the international association for the study of lung cancer, and the association for molecular pathology. J Thorac Oncol 2018;13:323-58.

3. Planchard D, Smit EF, Groen HJM, et al. Dabrafenib plus trametinib in patients with previously untreated BRAF(V600E)-mutant metastatic non-small-cell lung cancer: an open-label, phase 2 trial. Lancet Oncol 2017;18:1307-16.

4. Hanna NH, Schneider BJ, Temin SB, et al. Therapy for stage IV non-small-cell lung cancer without driver alterations: $\mathrm{ASCO}$ and $\mathrm{OH}(\mathrm{CCO})$ joint guideline update. J Clin Oncol 2020;38:1608-32.

5. Santarpia M, Aguilar A, Chaib I, et al. Non-Small-Cell Lung Cancer Signaling Pathways, Metabolism, and PD-1/ PD-L1 Antibodies. Cancers (Basel) 2020;12:1475.

6. Comoglio PM, Trusolino L, Boccaccio C. Known and novel roles of the MET oncogene in cancer: a coherent approach to targeted therapy. Nat Rev Cancer 2018;18:341-58.

7. Cortot AB, Kherrouche Z, Descarpentries C, et al. Exon 14 deleted MET receptor as a new biomarker and target in cancers. J Natl Cancer Inst 2017;109:djw262.

8. Frampton GM, Ali SM, Rosenzweig M, et al. Activation of MET via diverse exon 14 splicing alterations occurs in multiple tumor types and confers clinical sensitivity to MET inhibitors. Cancer Discov 2015;5:850-9.

9. Lipson D, Capelletti M, Yelensky R, et al. Identification of new ALK and RET gene fusions from colorectal and lung cancer biopsies. Nat Med 2012;18:382-4.

10. Drilon A, Hu ZI, Lai GGY, et al. Targeting RET-driven cancers: lessons from evolving preclinical and clinical landscapes. Nat Rev Clin Oncol 2018;15:151-67

11. Stinchcombe TE. Current management of RET rearranged non-small cell lung cancer. Ther Adv Med Oncol 2020;12:1758835920928634.

12. Vaishnavi A, Capelletti M, Le AT, et al. Oncogenic and drug-sensitive NTRK1 rearrangements in lung cancer. Nat Med 2013;19:1469-72.

13. Drilon A. TRK inhibitors in TRK fusion-positive cancers. Ann Oncol 2019;30:viii23-viii30.

14. Cooper CS, Park M, Blair DG, et al. Molecular cloning of a new transforming gene from a chemically transformed human cell line. Nature 1984;311:29-33.

15. Park M, Dean M, Cooper CS, et al. Mechanism of met oncogene activation. Cell 1986;45:895-904.

16. Dean M, Park M, Le Beau MM, et al. The human met oncogene is related to the tyrosine kinase oncogenes. Nature 1985;318:385-8.

17. Giordano S, Ponzetto C, Di Renzo MF, et al. Tyrosine kinase receptor indistinguishable from the c-met protein. Nature 1989;339:155-6.

18. Naldini L, Weidner KM, Vigna E, et al. Scatter factor and hepatocyte growth factor are indistinguishable ligands for the MET receptor. EMBO J 1991;10:2867-78.

19. Zhang J, Babic A. Regulation of the MET oncogene: molecular mechanisms. Carcinogenesis 2016;37:345-55.

20. Giordano S, Di Renzo MF, Narsimhan RP, et al. Biosynthesis of the protein encoded by the c-met protooncogene. Oncogene 1989;4:1383-8.

21. Gherardi E, Youles ME, Miguel RN, et al. Functional map and domain structure of MET, the product of the c-met protooncogene and receptor for hepatocyte growth factor/ scatter factor. Proc Natl Acad Sci 2003;100:12039-44.

22. Bottaro DP, Rubin JS, Faletto DL, et al. Identification of the hepatocyte growth factor receptor as the c-met protooncogene product. Science 1991;251:802-4.

23. Naldini L, Vigna E, Narsimhan RP, et al. Hepatocyte growth factor (HGF) stimulates the tyrosine kinase activity of the receptor encoded by the proto-oncogene c-MET. Oncogene 1991;6:501-4.

24. Gherardi E, Birchmeier W, Birchmeier C, et al. Targeting MET in cancer: rationale and progress. Nat Rev Cancer 2012;12:89-103.

25. Ponzetto C, Bardelli A, Zhen Z, et al. A multifunctional docking site mediates signaling and transformation by the hepatocyte growth factor/scatter factor receptor family. Cell 1994;77:261-71.

26. Viticchiè G, Muller PAJ. c-Met and other cell surface molecules: interaction, activation and functional consequences. Biomedicines 2015;3:46-70.

27. Peschard P, Fournier TM, Lamorte L, et al. Mutation of the $\mathrm{c}-\mathrm{Cbl}$ TKB domain binding site on the Met receptor tyrosine kinase converts it into a transforming protein. Mol Cell 2001;8:995-1004.

28. Petrelli A, Gilestro GF, Lanzardo S, et al. The endophilinCIN85-Cbl complex mediates ligand-dependent downregulation of c-Met. Nature 2002;416:187-90.

29. Joffre C, Barrow R, Ménard L, et al. A direct role for Met endocytosis in tumorigenesis. Nature Cell Biol 2011;13:827-37.

30. Foveau B, Ancot F, Leroy C, et al. Down-regulation of the met receptor tyrosine kinase by presenilin-dependent 
regulated intramembrane proteolysis. Mol Biol Cell 2009;20:2495-507.

31. Boccaccio C, Comoglio PM. Invasive growth: a METdriven genetic programme for cancer and stem cells. Nat Rev Cancer 2006;6:637-45.

32. Koch JP, Aebersold DM, Zimmer Y, et al. MET targeting: time for a rematch. Oncogene 2020;39:2845-62.

33. Ma PC, Tretiakova MS, MacKinnon AC, et al. Expression and mutational analysis of MET in human solid cancers. Genes Chromosomes Cancer 2008;47:1025-37.

34. Ichimura E, Maeshima A, Nakajima T, et al. Expression of c-met/HGF receptor in human nonsmall cell lung carcinomas in vitro and in vivo and its prognostic significance. Jpn J Cancer Res 1996;87:1063-9.

35. Park S, Choi YL, Sung CO, et al. High MET copy number and MET overexpression: Poor outcome in non-small cell lung cancer patients. Histol Histopathol 2012;27:197-207.

36. Al-Saad S, Richardsen E, Kilvaer TK, et al. The impact of MET, IGF-1, IGF1R expression and EGFR mutations on survival of patients with non-small-cell lung cancer. PLoS One 2017;12:e0181527.

37. Santarpia M, Gil N, Rosell R. Strategies to overcome resistance to tyrosine kinase inhibitors in non-small-cell lung cancer. Expert Rev Clin Pharmacol 2015;8:461-77.

38. Lazzari C, Gregorc V, Karachaliou N, et al. Mechanisms of resistance to osimertinib. J Thorac Dis 2020;12:2851-8.

39. Ma PC, Kijima T, Maulik G, et al. c-MET mutational analysis in small cell lung cancer: novel juxtamembrane domain mutations regulating cytoskeletal functions. Cancer Res 2003;63:6272-81.

40. Krishnaswamy S, Kanteti R, Duke-Cohan JS, et al. Ethnic differences and functional analysis of MET mutations in lung cancer. Clin Cancer Res 2009; 15:5714-23.

41. Shieh JM, Tang YA, Yang TH, et al. Lack of association of C-Met-N375S sequence variant with lung cancer susceptibility and prognosis. Int J Med Sci 2013;10:988-94.

42. Tyner JW, Fletcher LB, Wang EQ, et al. MET receptor sequence variants R970C and T992I lack transforming capacity. Cancer Res 2010;70:6233-7.

43. Stransky N, Cerami E, Schalm S, et al. The landscape of kinase fusions in cancer. Nat Commun 2014;5:4846.

44. Plenker D, Bertrand M, de Langen AJ, et al. Structural alterations of MET trigger response to MET kinase inhibition in lung adenocarcinoma patients. Clin Cancer Res 2018;24:1337-43.

45. Davies KD, Ng TL, Estrada-Bernal A, et al. Dramatic response to crizotinib in a patient with lung cancer positive for an HLA-DRB1-MET gene fusion. JCO Precis Oncol 2017;2017:1-6.

46. Zhu YC, Wang WX, Xu CW, et al. Identification of a novel crizotinib-sensitive MET-ATXN7L1 gene fusion variant in lung adenocarcinoma by next generation sequencing. Ann Oncol 2018;29:2392-3.

47. Pan Y, Zhang Y, Ye T, et al. Detection of Novel NRG1, EGFR, and MET Fusions in Lung Adenocarcinomas in the Chinese Population. J Thorac Oncol 2019;14:2003-8.

48. Ma PC, Jagadeeswaran R, Jagadeesh S, et al. Functional expression and mutations of c-Met and its therapeutic inhibition with SU11274 and small interfering RNA in non- small cell lung cancer. Cancer Res 2005;65:1479-88.

49. Kong-Beltran M, Seshageri S, Zha J, et al. Somatic mutations lead to an oncogenic deletion of met in lung cancer. Cancer Res 2006;66:283-9.

50. Paik PK, Drilon A, Fan PD, et al. Response to MET inhibitors in patients with stage IV lung adenocarcinomas harboring MET mutations causing exon 14 skipping. Cancer Discov 2015;5:842-9.

51. Vigna E, Gramaglia D, Longati P, et al. Loss of the exon encoding the juxtamembrane domain is essential for the oncogenic activation of TPR-MET. Oncogene 1999;18:4275-81.

52. Cancer Genome Atlas Research Network. Comprehensive molecular profiling of lung adenocarcinoma. Nature 2014;511:543-50.

53. Awad MM, Oxnard GR, Jackman DM, et al. MET exon 14 mutations in non-small-cell lung cancer are associated with advanced age and stage-dependent MET genomic amplification and c-Met overexpression. J Clin Oncol 2016;34:721-30.

54. Tong JH, Yeung SF, Chan AWH, et al. MET amplification and exon 14 splice site mutation define unique molecular subgroups of non- small cell lung carcinoma with poor prognosis. Clin Cancer Res 2016;22:3048-56.

55. Liu X, Jia Y, Stoopleret MB, et al. Next-generation sequencing of pulmonary sarcomatoid carcinoma reveals high frequency of actionable MET Gene Mutations. J Clin Oncol 2016;34:794-802.

56. Schrock AB, Frampton GM, Suh J, et al. Characterization of 298 patients with lung cancer harboring MET exon 14 skipping alterations. J Thorac Oncol 2016;11:1493-502.

57. Zheng D, Wang R, Ye T, et al. MET exon 14 skipping defines a unique molecular class of non-small cell lung cancer. Oncotarget 2016;7:41691-702.

58. Vuong HG, Ho ATN, Altibi AMA, et al. Clinicopathological implications of MET exon 14 
mutations in non-small cell lung cancer - A systematic review and meta-analysis. Lung Cancer 2018;123:76-82.

59. Kim EK, Kim KA, Lee CY, et al. Molecular Diagnostic Assays and Clinicopathologic Implications of MET Exon 14 Skipping Mutation in Non-small-cell Lung Cancer. Clin Lung Cancer 2019;20:e123-e132.

60. Pruis MA, Geurts-Giele WRR, von der TJH, et al. Highly accurate DNA-based detection and treatment results of MET exon 14 skipping mutations in lung cancer. Lung Cancer 2020;140:46-54.

61. Davies KD, Lomboy A, Lawrence CA, et al. DNA-Based versus RNA-Based Detection of MET Exon 14 Skipping Events in Lung Cancer. J Thorac Oncol 2019;14:737-41.

62. Awad MM, Lee JK, Madison R, et al. Characterization of 1,387 NSCLCs with MET exon 14 (METex14) skipping alterations (SA) and potential acquired resistance (AR) mechanisms. J Clin Oncol 2020;38:Abstract 9511.

63. Benvenuti S, Gentile A, Lazzari L, et al. An 'in-cell trial' to assess the efficacy of a monovalent anti-MET antibody as monotherapy and in association with standard cytotoxics. Mol Oncol 2014;8:378-88.

64. Scagliotti G, von Pawel J, Novello S, et al. Phase III multinational, randomized, double- blind, placebocontrolled study of tivantinib (ARQ 197) plus erlotinib versus erlotinib alone in previously treated patients with locally advanced or metastatic nonsquamous non-smallcell lung cancer. J Clin Oncol 2015;33:2667-74.

65. Spigel DR, Edelman MJ, O'Byrne K, et al. Results from the phase III randomized trial of onartuzumab plus erlotinib versus erlotinib in previously treated stage IIIB or IV non-small-cell lung cancer: METLung. J Clin Oncol 2017;35:412-20.

66. Cui JJ. Targeting Receptor Tyrosine Kinase MET in Cancer: Small Molecule Inhibitors and Clinical Progress. J Med Chem 2014;57:4427-53.

67. Shaw AT, Kim DW, Nakagawa K, et al. Crizotinib versus chemotherapy in advanced ALK-positive lung cancer. N Engl J Med 2013;368:2385-94.

68. Solomon BJ, Mok T, Kim DW, et al. First-line crizotinib versus chemotherapy in ALK-positive lung cancer. N Engl J Med 2014;371:2167-77.

69. Shaw AT, Ou SI, Bang Y, et al. Crizotinib in ROS1rearranged non small cell lung cancer. N Engl J Med 2014;371:1963-71.

70. Waqar SN, Morgensztern D, Sehn J. MET mutation associated with responsiveness to crizotinib. J Thorac Oncol 2015;10:e29-e31.

71. Mendenhall MA, Goldman JW. MET- Mutated NSCLC with Major Response to Crizotinib. J Thorac Oncol 2015;10:e33-e34.

72. Mahjoubi L, Gazzah A, Besse B, et al. A never- smoker lung adenocarcinoma patient with a MET exon 14 mutation $(\mathrm{D} 1028 \mathrm{~N})$ and a rapid partial response after crizotinib. Invest. New Drugs 2016;34:397-8.

73. Jorge SE, Schulman S, Freed JA, et al. Responses to the multitargeted MET/ALK/ROS1 inhibitor crizotinib and cooccurring mutations in lung adenocarcinomas with MET amplification or MET exon 14 skipping mutation. Lung Cancer 2015;90:369-74.

74. Jenkins RW, Oxanrd GR, Elkin S, et al. Response to crizotinib in a patient with lung adenocarcinoma harboring a MET splice site mutation. Clin. Lung Cancer 2015;16:e101-e104.

75. Drilon AE, Camidge DR, Ou SHI, et al. Efficacy and safety of crizotinib in patients (pts) with advanced MET exon 14-altered no-nsmall cell lung cancer (NSCLC). J Clin Oncol 2016;34:108.

76. Drilon A, Clark JW, Weiss J, et al. Antitumor activity of crizotinib in lung cancers harboring a MET exon 14 alteration. Nat Med 2020;26:47-51.

77. Moro-Sibilot D, Cozic N, Perol M, et al. Crizotinib in c-MET- or ROS1-positive NSCLC: results of the AcSe phase II trial. Ann Oncol 2019;30:1985-91.

78. Landi L, Chiari R, Tiseo M, et al. Crizotinib in MET deregulated or ROS1 rearranged pretreated non-small-cell lung cancer (METROS): a phase II, prospective, multicentre, two-arms trial. Clin Cancer Res 2019;25:7312-9.

79. Vuong HG, Nguyen TQ, Nguyen HC, et al. Efficacy and Safety of Crizotinib in the Treatment of Advanced NonSmall-Cell Lung Cancer with ROS1 Rearrangement or MET Alteration: A Systematic Review and Meta-Analysis. Target Oncol 2020;15:589-98.

80. Cometriq: FDA prescribing information. Available online: https://www.accessdata.fda.gov/drugsatfda_docs/ label/2020/203756s008lbl.pdf. Revised: 1/2020.

81. Cabometyx: FDA prescribing information. Available online: https://www.accessdata.fda.gov/drugsatfda_docs/ label/2019/208692s0031bl.pdf. Revised: 1/2019.

82. Yakes FM, Chen J, Tan J, et al. Cabozantinib (XL184), a novel MET andVEGFR2 inhibitor, simultaneously suppresses metastasis, angiogenesis, andtumor growth, Mol Cancer Ther 2011;10:2298-308.

83. Neal JW, Dahlberg SE, Wakelee HA, et al. Erlotinib, cabozantinib, or erlotinib plus cabozantinib as secondline or third-line treatment of patients with EGFR wild- 
type advanced non-small-cell lung cancer (ECOG-ACRIN 1512): a randomised, controlled, open-label, multicentre, Phase 2 trial. Lancet Oncol 2016;17:1661-71.

84. Reckamp KL, Frankel PH, Ruel N, et al. Phase II Trial of Cabozantinib Plus Erlotinib in Patients With Advanced Epidermal Growth Factor Receptor (EGFR)-Mutant Non-small Cell Lung Cancer With Progressive Disease on Epidermal Growth Factor Receptor Tyrosine Kinase Inhibitor Therapy: A California Cancer Consortium Phase II Trial (NCI 9303). Front Oncol 2019;9:132.

85. Klempner SJ, Borghei A, Hakimian B, et al. Intracranial activity of cabozantinib in MET exon 14-positive NSCLC with brain metastases. J Thorac Oncol 2017;12:152-6.

86. Wang SXY, Zhang BM, Wakelee HA, et al. Case series of MET exon 14 skipping mutation-positive non-small-cell lung cancers with response to crizotinib and cabozantinib. Anticancer Drugs 2019;30:537-41.

87. Yan SB, Peek VL, Ajamie R, et al. LY2801653 is an orally bioavailable multi-kinase inhibitor with potent activity against MET, MST1R, and other oncoproteins, and displays anti-tumor activities in mouse xenograft models. Invest New Drugs 2013;31:833-44.

88. He AR, Cohen RB, Denlinger CS, et al. First-inhuman phase I study of merestinib, an oral multikinase inhibitor, in patients with advanced cancer. Oncologist 2019;24:e930-e942.

89. $\mathrm{Wu} \mathrm{W}, \mathrm{Bi} \mathrm{C}$, Credille KM, et al. Inhibition of tumor growth and metastasis in non-small cell lung cancer by LY2801653, an inhibitor of several oncokinases, including MET. Clin Cancer Res 2013;19:5699-710.

90. Kawada I, Hasina R, Arif Q, et al. Dramatic antitumor effects of the dual MET/RON small-molecule inhibitor LY2801653 in non-small cell lung cancer. Cancer Res 2014;74:884-95.

91. Yan SB, Um SL, Peek VL, et al. MET-targeting antibody (emibetuzumab) and kinase inhibitor (merestinib) as single agent or in combination in a cancer model bearing MET exon 14 skipping. Invest New Drugs 2018;36:536-44.

92. Engstrom LD, Aranda R, Lee M, et al. Glesatinib exhibits antitumor activity in lung cancer models and patients harboring MET exon 14 mutations and overcomes mutation-mediated resistance to type I MET inhibitors in nonclinical models. Clin Cancer Res 2017;23:6661-72.

93. Bazhenova L, Kim D, Cavanna L, et al. P2.06-017 Amethyst NSCLC trial: phase 2 study of MGCD265 in patients with advanced or metastatic NSCLC with activating genetic alterations in MET. J Thorac Oncol 2017;12:S1080-1.
94. Der-Torossian H, Shapiro G, Janne PA, et al. MET mutant allele frequency (MAF) is correlated with glesatinib anti-tumor activity in patients with advanced non-small cell lung cancer (NSCLC) harboring MET alterations. Eur J Cancer 2018;103:e133.

95. Liu X, Wang Q, Yang G, et al. A novel kinase inhibitor, INCB28060, blocks c-MET-dependent signaling, neoplastic activities, and cross-talk with EGFR and HER3. Clin Cancer Res 2011;17:7127-38.

96. Baltschukat S, Engstler BS, Huang A, et al. Capmatinib (INC280) Is Active Against Models of Non-Small Cell Lung Cancer and Other Cancer Types with Defined Mechanisms of MET Activation. Clin Cancer Res 2019;25:3164-75

97. Schuler M, Berardi R, Lim WT, et al. Molecular correlates of response to capmatinib in advanced non-small-cell lung cancer: clinical and biomarker results from a phase I trial. Ann Oncol 2020;31:789-97.

98. Wolf J, Seto T, Han JY, et al. Capmatinib (INC280) in MET ex14-mutated advanced non-small cell lung cancer (NSCLC): Efficacy data from the phase II GEOMETRY mono-1 study. Presented at the American Society of Clinical Oncology (ASCO) Annual Meeting, Chicago, Illinois. J Clin Oncol 2019;37:Abstract 9004.

99. Wolf J, Seto T, Han JY, et al. Capmatinib in MET Exon 14-Mutated or MET-Amplified Non-Small-Cell Lung Cancer. N Engl J Med 2020;383:944-57.

100. Bladt F, Faden B, Friese-Hamim M, et al. EMD 1214063 and EMD 1204831 constitute a new class of potent and highly selective c-Met inhibitors. Clin Cancer Res 2013;19:2941-51.

101. Falchook GS, Kurzrock R, Amin HM, et al. First-in-man phase I trial of the selective MET inhibitor tepotinib in patients with advanced solid tumors. Clin Cancer Res 2020;26:1237-46.

102.Paik PK, Felip E, Veillon R, et al. Tepotinib in NonSmall-Cell Lung Cancer with MET Exon 14 Skipping Mutations. N Engl J Med 2020;383:931-43.

103. Markham A. Tepotinib: First Approval. Drugs 2020;80:829-33.

104. Wu YL, Cheng Y, Zhou J, et al. Tepotinib plus gefitinib in patients with EGFR-mutant non-small-cell lung cancer with MET overexpression or MET amplification and acquired resistance to previous EGFR inhibitor (INSIGHT study): an open-label, phase 1b/2, multicentre, randomised trial. Lancet Respir Med 2020;8:1132-43.

105. Rosell R, Chaib I, Santarpia M. Targeting MET amplification in EGFR-mutant non-small-cell lung cancer. 
Lancet Respir Med 2020;8:1068-70.

106. Gu Y, Sai Y, Wang J, et al. Preclinical pharmacokinetics, disposition, and translational pharmacokinetic/ pharmacodynamic modeling of savolitinib, a novel selective cMet inhibitor. Eur J Pharm Sci 2019;136:104938.

107. Oxnard GR, Yang JC, Yu H, et al. TATTON: a multi-arm, phase Ib trial of osimertinib combined with selumetinib, savolitinib, or durvalumab in EGFR-mutant lung cancer. Ann Oncol 2020;31:507-16.

108. Barry E, Henry R, Borodovsky A, et al. Activity of Savolitinib against MET Ex14 mutations and resistance to METi through decoupling from MYC expression. Presented at the American Association of Cancer Research (AACR) Annual Meeting, New Orleans, Louisiana, USA, 16-20 April 2016. Cancer Res 2016;76:14.

109. Han S, Fang J, Lu S, et al. Response and acquired resistance to savolitinib in a patient with pulmonary sarcomatoid carcinoma harboring MET exon 14 skipping mutation: a case report. Onco Targets Ther 2019;12:7323-28.

110.Lu S, Fang J, Li X, et al. Phase II study of savolitinib in patients (pts) with pulmonary sarcomatoid carcinoma (PSC) and other types of non-small cell lung cancer (NSCLC) harboring MET exon 14 skipping mutations (METex14+). J Clin Oncol 2020;38:Abstract 9519.

111.Camidge DR, Janku F, Martinez-Bueno A, et al. Safety and preliminary clinical activity of the MET antibody mixture, Sym015 in advanced non-small cell lung cancer (NSCLC) patients with MET amplification/exon 14 deletion (METAmp/Ex14A). J Clin Oncol 2020;38:Abstract 9510.

112. Mazieres J, Drilon A, Lusque A, et al. Immune checkpoint inhibitors for patients with advanced lung cancer and oncogenic driver alterations: results from the IMMUNOTARGET registry. Ann Oncol 2019;30:1321-8.

113. Sabari JK, Leonardi GC, Shu CA, et al. PD-L1 expression, tumor mutational burden, and response to immunotherapy in patients with MET exon 14 altered lung cancers. Ann Oncol 2018;29:2085-91.

114. Reis H, Metzenmacher M, Goetz M, et al. MET Expression in Advanced Non-Small-Cell Lung Cancer: Effect on Clinical Outcomes of Chemotherapy, Targeted Therapy, and Immunotherapy. Clin Lung Cancer 2018;19:e441-e463.

115. Mayenga M, Assié JB, Monnet I, et al. Durable responses to immunotherapy of non-small cell lung cancers harboring MET exon-14-skipping mutation: A series of 6 cases. Lung Cancer 2020;150:21-5.

116. Recondo G, Che J, Jänne PA, et al. Targeting MET Dysregulation in Cancer. Cancer Discov 2020;10:922-34.
117. Bahcall M, Sim T, Paweletz CP, et al. Acquired METD $1228 \mathrm{~V}$ mutation and resistance to MET inhibition in lung cancer. Cancer Discov 2016;6:1334-41.

118. Dong HJ, Li P, Wu C, et al. Response and acquired resistance to crizotinib in Chinese patients with lung adenocarcinomas harboring MET exon 14 splicing alternations. Lung Cancer 2016;102:118-21.

119. Heist RS, Sequist LV, Borger D, et al. Acquired resistance to crizotinib in NSCLC with MET exon 14 skipping. J Thorac Oncol 2016;11:1242-5.

120. Ou SI, Young L, Schrock AB, et al. Emergence of preexisting MET Y1230C mutation as a resistance mechanism to crizotinib in NSCLC with MET exon 14 skipping. J Thorac Oncol 2017;12:137-40.

121.Zhang Y, Yin J, Peng F. Acquired resistance to crizotinib in advanced lung adenocarcinoma with MET exon 14 skipping. Lung Cancer 2017;113:69-71.

122. Schrock AB, Lai A, Ali SM, et al. Mutation of MET $\mathrm{Y} 1230$ as an acquired mechanism of crizotinib resistance in NSCLC with MET exon 14 skipping. J Thorac Oncol 2017;12:e89-90.

123. Guo R, Offin M, Brannon R, et al. MET inhibitor resistance in patients with MET exon 14-altered lung cancers. J Clin Oncol 2019;37:Abstract 9006.

124.Jin W, Shan B, Liu H, et al. Acquired mechanism of crizotinib resistance in NSCLC with MET exon 14 skipping. J Thorac Oncol 2019;14:e137-9.

125. Fujino T, Kobayashi Y, Suda K, et al. Sensitivity and Resistance of MET exon 14 mutations in lung cancer to eight MET tyrosine kinase inhibitors in vitro. J Thorac Oncol 2019;14:1753-65.

126. Bahcall M, Awad MM, Sholl LM, et al. Amplification of wild-type KRAS imparts resistance to crizotinib in MET exon 14 mutant non-small cell lung cancer. Clin Cancer Res 2018;24:5963-76.

127. Suzawa K, Offin M, Lu D, et al. Activation of KRAS mediates resistance to targeted therapy in MET exon 14-mutant non-small cell lung cancer. Clin Cancer Res 2019;25:1248-60.

128. Recondo G, Bahcall M, Spurr LF, et al. Molecular Mechanisms of Acquired Resistance to MET Tyrosine Kinase Inhibitors in Patients with MET Exon 14-Mutant NSCLC. Clin Cancer Res 2020;26:2615-25.

129. An N, Xiong Y, LaRue AC, et al. Activation of Pim Kinases Is Sufficient to Promote Resistance to MET Small-Molecule Inhibitors. Cancer Res 2015;75:5318-28.

130.Jamme P, Fernandes M, Copin MC, et al. Alterations in the PI3K Pathway Drive Resistance to MET Inhibitors in 
NSCLC Harboring MET Exon 14 Skipping Mutations. J Thorac Oncol 2020;15:741-51.

131. Rotow JK, Gui P, Wu W, et al. Co-occurring Alterations in the RAS-MAPK Pathway Limit Response to MET Inhibitor Treatment in MET Exon 14 Skipping MutationPositive Lung Cancer. Clin Cancer Res 2020;26:439-49.

132. Guo T, Zhao S, Wang P, et al. YB-1 regulates tumor growth by promoting MACC1/c-MET pathway in human lung adenocarcinoma. Oncotarget 2017;8:48110-25.

133. Nilsson MB, Sun H, Robichaux J, et al. The YAP/

FOXM1 axis regulates EMT associated EGFR tyrosine

Cite this article as: Santarpia M, Massafra M, Gebbia V, D'Aquino A, Garipoli C, Altavilla G, Rosell R. A narrative review of MET inhibitors in non-small cell lung cancer with MET exon 14 skipping mutations. Transl Lung Cancer Res 2021;10(3):1536-1556. doi: 10.21037/tlcr-20-1113 kinase inhibitor resistance and increased expression of spindle assembly checkpoint components. J Thorac Oncol 2020;15:S11.

134. Sugano T, Seike M, Noro R, et al. Inhibition of ABCB1 Overcomes Cancer Stem Cell-like Properties and Acquired Resistance to MET Inhibitors in Non-Small Cell Lung Cancer. Mol Cancer Ther 2015;14:2433-40.

135.Awad MM, Leonardi GC, Kravets S, et al. Impact of MET inhibitors on survival among patients (pts) with MET exon 14 mutant (METdel14) non-small cell lung cancer (NSCLC). Lung Cancer 2019;133:96-102. 


\section{Key search}

We performed a literature search in PubMed, Scopus and Web of Science, for papers published up to September 2020 on MET in non-small lung cancer using the following terms: "MET", "MET deregulation", "MET and lung cancer", "MET exon 14 skipping mutations", "MET inhibitors". We also searched for abstracts on main international cancer congresses (ESMO, ASCO, IASLC) websites. We collected and reviewed data of both completed and ongoing studies.

Our search found several review articles about the role of MET in lung cancer and randomised trials on MET inhibitors in non-small cell lung cancer patients. We focused on results from studies with MET tyrosine kinase inhibitors (TKIs), including multitarget TKIs (crizotinib, cabozantinib, merestinib and glesatinib) and selective MET TKIs (capmatinib, tepotinib and savolitinib), in NSCLC patients with MET exon 14 skipping mutations.

The added value of this study is that we review very recent data from both published and ongoing clinical trials. We also provide an overview of most recently identified mechanisms of resistance to MET TKIs, potential therapeutic approaches to overcome resistance and recent evidence about the use of immunotherapy in this molecularly-defined subgroup of patients. We underline the emerging role of MET exon 14 skipping mutation as key oncogene and the importance of routine testing NSCLC tumor samples (tissue and/or ctDNA) for MET alterations. 